EPJ Web of Conferences 49, 05003 (2013)

DOI: $10.1051 /$ epjconf/20134905003

(C) Owned by the authors, published by EDP Sciences, 2013

\title{
Searches for new physics at CMS and ATLAS without Leptons or Resonances
}

\author{
Daniel Duggan ${ }^{1, \mathrm{a}}$ and Amitabh Lath ${ }^{1, \mathrm{~b}}$ \\ ${ }^{1}$ Rutgers University
}

\begin{abstract}
There is a long history of searches for new physics using resonances or leptons in the final state. We present searches for new physics that may have neither leptons or resonances in the final state. In these cases, the searches must be model driven. Examples include models of Dark Matter, Large Extra Dimensions, long lived particle, Magnetic Monopoles or Microscopic Black Holes. The analyses presented were performed at the ATLAS and CMS experiments at the Large Hadron Collider.
\end{abstract}

\section{Introduction}

We present new results from searches for new physics signatures performed by both the ATLAS [1] and CMS [2] Collaborations that might result in no leptons or resonance features in the final state. Such phenomena would be very difficult to extract from the data of a hadron collider. However, armed with specific models of new physics we have successfully performed searches for several models of new physics. These include Dark Matter, which couples to matter very weakly, but if produced, can be detected by Initial State Radiation (ISR) of a photon or a jet. Other models of non-leptonic, non-resonant signatures are long lived or stable particles such as those from hidden valley models, microscopic black holes, and magnetic monopoles.

\section{Dark Matter and LED}

Models of Dark Matter (DM) make compelling targets for searches for new physics. The existence of DM has been confirmed by astronomy, and there is a reasonable chance that the DM will be accessible at hadron colliders.

However, even if DM is produced in colliders, its coupling to Standard Model (SM) particles may be small enough so that it leaves no trace in the detector. We present DM searches that look for large missing transverse energy (MET) and either an associated jet or photon. In the monojet search, the jet itself comes from ISR with large transverse momentum. The CMS search [3] selects events with MET $>350 \mathrm{GeV}$ and a jet with $\mathrm{p}_{\mathrm{T}}>110 \mathrm{GeV}$, collected in the 2011 run of the LHC at $\sqrt{s}=7 \mathrm{TeV}$. Similarly, the ATLAS study [4] probes the same event topology with requirements of MET $>120 \mathrm{GeV}$ and a jet $\mathrm{p}_{\mathrm{T}}>120 \mathrm{GeV}$, using $10 \mathrm{fb}^{-1}$ of luminosity, collected during 2012 at $\sqrt{s}=8 \mathrm{TeV}$.

\footnotetext{
a e-mail: duggan@cern.ch

be-mail: lath@physics.rutgers.edu
}

Large Extra Dimensions (LED) models also lead to even signatures with large MET. Figure 1 shows the jet $\mathrm{p}_{\mathrm{T}}$ and MET distributions for data, several backgrounds, as well as specific DM and LED models.

We see no deviation from SM expectations, and set limits on DM mass and coupling. We compare our results to those from dedicated DM searches such as CDMS [5], COUPP [6], IceCube [7] and Super Kamiodande [8]. Figure 2 shows the limits on DM production cross section versus mass, as well as limits set on LED for both the CMS and ATLAS monojet searches.

\section{Long Lived Particles}

The Higgs sector of the SM is particularly sensitive to new physics. If the Higgs decays to long-lived neutral particles $\left(\pi_{v}\right)$ that subsequently decay with a large displaced vertex to heavy fermions, the signal may only show up in the muon chambers, past the calorimeter.

Both ATLAS and CMS have searched for long lived particles. The ATLAS analysis [9] searched for a light Higgs decay to a pair of long lived particles $\left(\pi_{v}\right)$ that decay inside the muon spectrometer using $1.94 \mathrm{fb}^{-1}$ of data collected in 2011 at $\sqrt{s}=7 \mathrm{TeV}$. The selection criteria required at least three muon candidates. The results were consistent with SM background and ruled out $c \tau$ in the range of $0.50 \mathrm{~m}$ to $20.65 \mathrm{~m}$ for $\mathrm{H}, \pi_{v}$ masses of 120 and $20 \mathrm{GeV}$ to $c \tau$ in the range of $1.10 \mathrm{~m}$ to $26.75 \mathrm{~m}$ for $\mathrm{H}, \pi_{v}$ masses of 140 and $50 \mathrm{GeV}$.

CMS searched for heavy stable charged particles (HSCP) using two techniques [10]. The first analysis used the inner tracking detectors to select a sample of events containing tracks with high momentum and high ionization energy loss. A second analysis collected a sample of events with high-momentum tracks satisfying muon identification requirements as well as having high-ionization and long time-of-flight. In both cases, the results are consistent with the expected background. A limit of 928 (626) $\mathrm{GeV}$ was set for a gluino (scalar top quark), and 

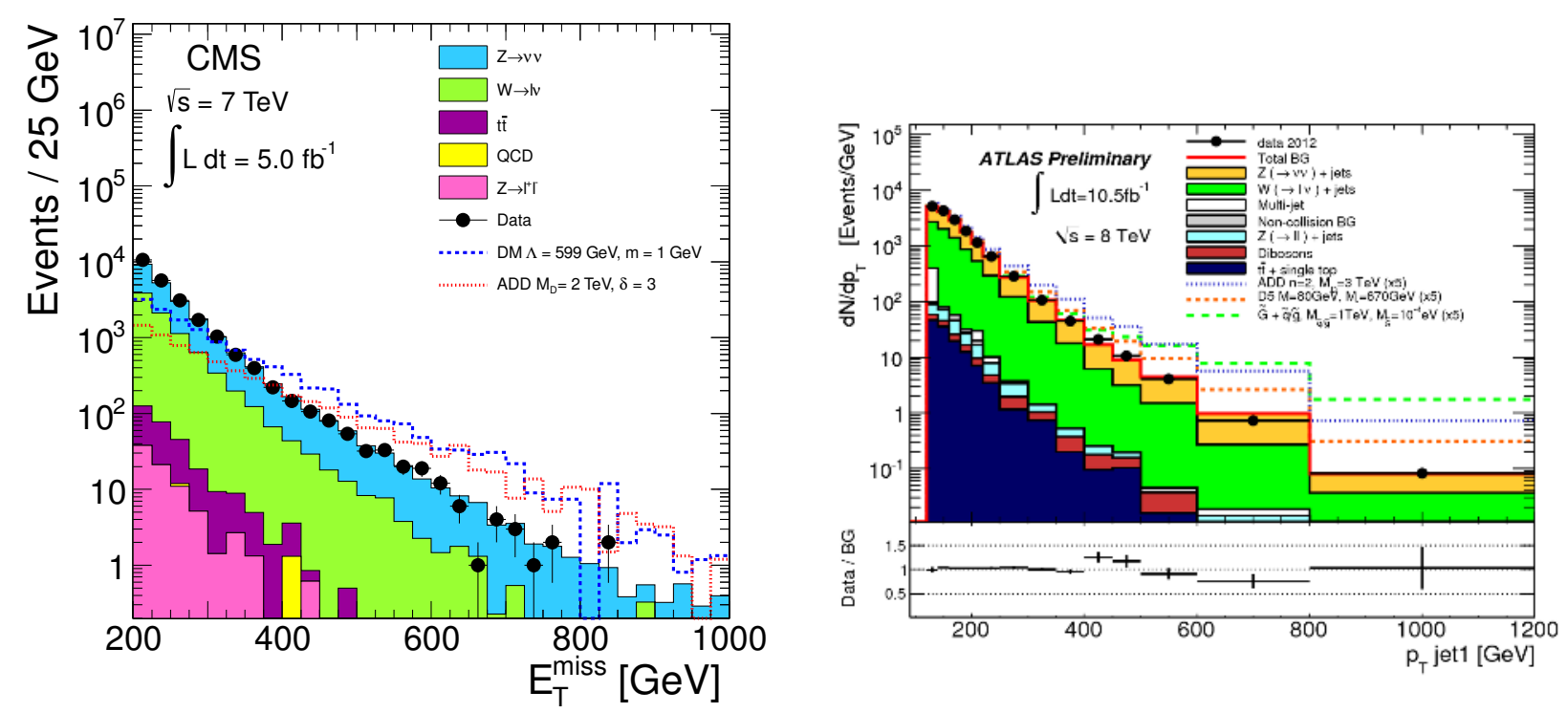

Figure 1. The MET and jet $\mathrm{p}_{\mathrm{T}}$ distributions from CMS (left) and ATLAS (right). The backgrounds and models of DM and LED are shown, along with data.
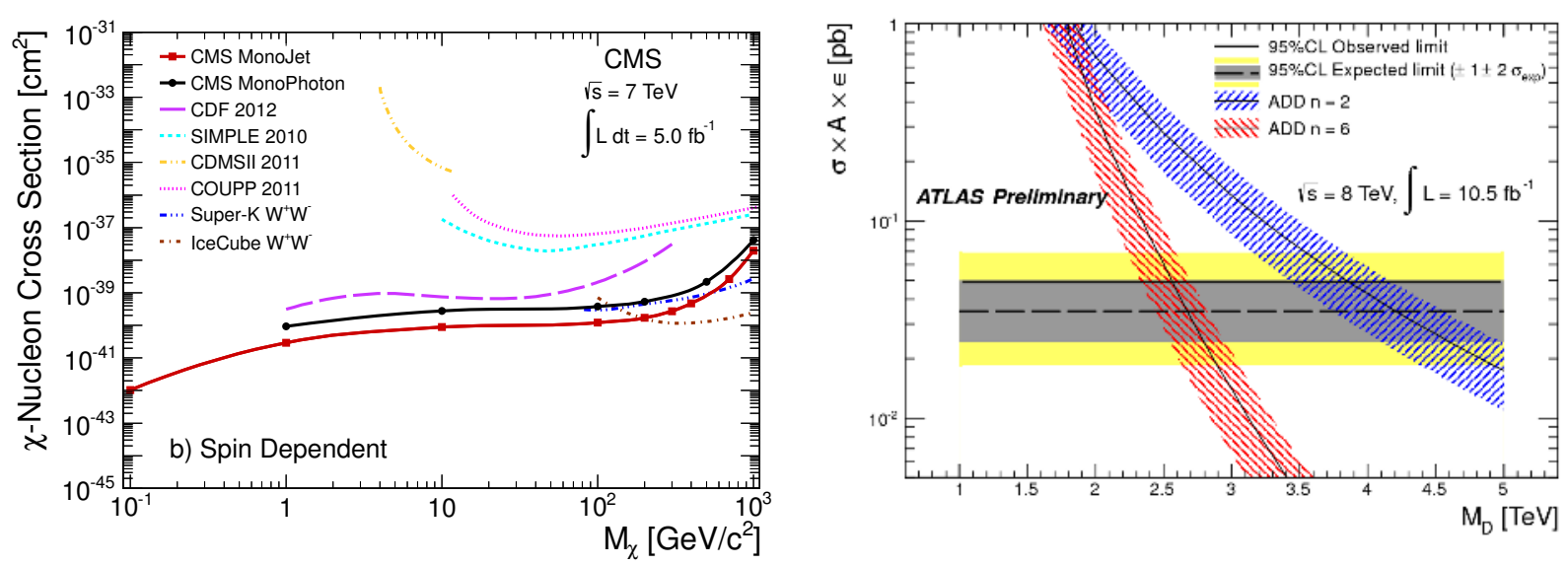

Figure 2. Limits on DM from CMS (left), and LED from ATLAS (right).

$223 \mathrm{GeV}$ for a pair produced scalar $\tau$. Mass limits for a hyper-kaon were placed at 484, 602, and $747 \mathrm{GeV}$ for hyper-r masses of 800,1200 , and $1600 \mathrm{GeV}$, respectively.

\section{Microscopic Black Holes}

A search for microscopic black holes (BH) [11] was conducted by CMS using $3.7 \mathrm{fb}^{-1}$ of data in 2012. Events with large total transverse energy $\left(\mathrm{S}_{\mathrm{T}}\right)$ were analyzed for the presence of multiple objects (jets, leptons, and photons). These are signals of evaporating semiclassical and quantum black holes, and string balls. Events with low multiplicity of objects were used to fit the background.

Figure 3 shows the $S_{T}$ distribution for the high object multiplicity events, along with background prediction and $\mathrm{BH}$ model expectations.

Agreement with the SM backgrounds, dominated by QCD, allowed setting limits on BH production. Model- independent limits were set on new physics processes producing high-multiplicity, energetic final states. In addition, new model-specific indicative limits are set excluding semiclassical black holes with masses below 4.1 to $6.1 \mathrm{TeV}$.

\section{Magnetic Monopoles}

Magnetic monopoles searches have been the subject of several searches since first being suggested by Dirac. They would restore the symmetry of electromagnetism and explain the quantization of electric charge.

ATLAS searched for mangetic monopoles using 2.0 $\mathrm{fb}^{-1}$ of data collected in 2011 at $\sqrt{s}=7 \mathrm{TeV}$ [12]. The analysis required narrow deposits in the EM calorimeter, along with high ionization in the tracker. The results agreed with the expectation of SM background, and were used to set a limit for monopole production, for Dirac 
monopole masses in the $200-1500 \mathrm{GeV}$, assuming full efficiency. The limits for Drell-Yan cross section production of monopole pairs is weaker, $95 \%$ confidence level varies from $145 \mathrm{fb}$ to $16 \mathrm{fb}$ for monopoles with mass between 200 and $1200 \mathrm{GeV}$.

\section{References}

[1] G. Aad et al. [ATLAS Collaboration], JINST 3, S08003 (2008).

[2] S. Chatrchyan et al. [CMS Collaboration], JINST 3, S08004 (2008).

[3] S. Chatrchyan et al. [CMS Collaboration], JHEP 1209, 094 (2012).

[4] [ATLAS Collaboration], ATLAS-CONF-2012-147.

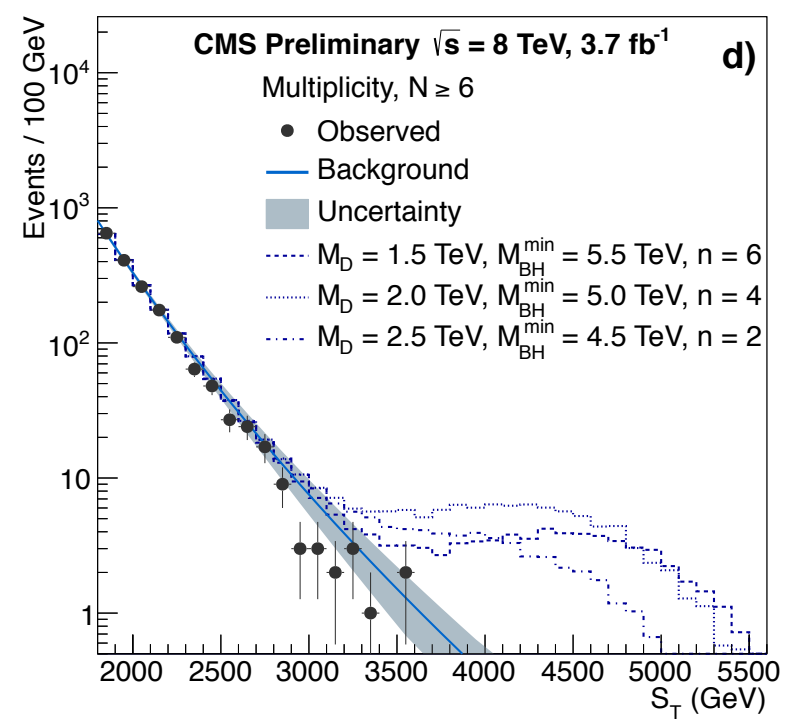

Figure 3. The $\mathrm{S}_{\mathrm{T}}$ distribution comparing data and background. The expectation from $\mathrm{BH}$ models are also shown.
[5] Z. Ahmed et al. [CDMS-II Collaboration], Phys. Rev. Lett. 106, 131302 (2011).

[6] E. Behnke, J. Behnke, S. J. Brice, D. Broemmelsiek, J. I. Collar, P. S. Cooper, M. Crisler and C. E. Dahl et al., Phys. Rev. Lett. 106, 021303 (2011).

[7] R. Abbasi et al. [IceCube Collaboration], Phys. Rev. D 85, 042002 (2012).

[8] T. Tanaka et al. [Super-Kamiokande Collaboration], Astrophys. J. 742, 78 (2011).

[9] G. Aad et al. [ATLAS Collaboration], Phys. Rev. Lett. 108, 251801 (2012).

[10] S. Chatrchyan et al. [CMS Collaboration], Phys. Lett. B 713, 408 (2012).

[11] [CMS Collaboration], CMS-PAS-EXO-12-009.

[12] G. Aad et al. [ATLAS Collaboration], Phys. Rev. Lett. 109, 261803 (2012). 